\title{
Minimal full polarized embeddings of dual polar spaces
}

\author{
Ilaria Cardinali · Bart De Bruyn · Antonio Pasini
}

Received: 26 June 2005 / Accepted: 20 April 2006 /

Published online: 11 July 2006

(C) Springer Science + Business Media, LLC 2007

\begin{abstract}
Let $\Delta$ be a thick dual polar space of rank $n \geq 2$ admitting a full polarized embedding $e$ in a finite-dimensional projective space $\Sigma$, i.e., for every point $x$ of $\Delta, e$ maps the set of points of $\Delta$ at non-maximal distance from $x$ into a hyperplane $e^{*}(x)$ of $\Sigma$. Using a result of Kasikova and Shult [11], we are able the show that there exists up to isomorphisms a unique full polarized embedding of $\Delta$ of minimal dimension. We also show that $e^{*}$ realizes a full polarized embedding of $\Delta$ into a subspace of the dual of $\Sigma$, and that $e^{*}$ is isomorphic to the minimal full polarized embedding of $\Delta$. In the final section, we will determine the minimal full polarized embeddings of the finite dual polar spaces $D Q(2 n, q), D Q^{-}(2 n+1, q), D H(2 n-$ $1, q^{2}$ ) and $D W(2 n-1, q)$ ( $q$ odd), but the latter only for $n \leq 5$. We shall prove that the minimal full polarized embeddings of $D Q(2 n, q), D Q^{-}(2 n+1, q)$ and $D H(2 n-$ $\left.1, q^{2}\right)$ are the 'natural' ones, whereas this is not always the case for $D W(2 n-1$, $q)$.
\end{abstract}

Keywords Dual polar space $\cdot$ Polarized embedding $\cdot$ Universal embedding

B. De Bruyn: Postdoctoral Fellow of the Research Foundation - Flanders.

I. Cardinali $(\bowtie) \cdot$ A. Pasini

Dipartimento di Scienze Matematiche e Informatiche "R. Magari", Università di Siena, Pian dei Mantellini, 44 I-53100 Siena, Italy

e-mail: cardinali3@unisi.it

A. Pasini

e-mail: pasini@unisi.it

B. De Bruyn

Department of Pure Mathematics and Computer Algebra, Ghent University, Galglaan, 2, B-9000

Gent, Belgium

e-mail: bdb@cage.ugent.be 


\section{Introduction}

\subsection{Basic terminology and notation}

Let $\Pi$ be a thick polar space of rank $n \geq 2$ and let $\Delta$ be its associated dual polar space, i.e., $\Delta$ is the point-line geometry with points, respectively lines, the maximal, respectively next-to-maximal, singular subspaces of $\Pi$ (natural incidence). If $x$ and $y$ are two points of $\Delta$, then $\mathrm{d}(x, y)$ denotes the distance between $x$ and $y$ in the collinearity graph of $\Delta$. If $x$ is a point of $\Delta$ and if $k \in \mathbb{N}$, then $\Delta_{k}(x)$ denotes the set of points at distance $k$ from $x$ and $x^{\perp}$ denotes the set of points equal to or collinear with $x$. If $X$ and $Y$ are nonempty sets of points, then $\mathrm{d}(X, Y)$ denotes the minimal distance between a point of $X$ and a point of $Y$. A nonempty set $X$ of points of $\Delta$ is called a subspace if every line containing two points of $X$ has all its points in $X$. A subspace $X$ is called convex if every point on a shortest path (in the collinearity graph) between two points of $X$ is also contained in $X$.

For every point $x$ of $\Delta$, let $H_{x}$ denote the set of points of $\Delta$ at non-maximal distance from $x$. Since $\Delta$ is a near polygon [15], $H_{x}$ is a hyperplane of $\Delta$, i.e. a proper subspace of $\Delta$ meeting each line. It is well-known that $H_{x}$ is a maximal subspace of $\Delta$, see e.g. [2, p. 156].

The convex subspaces of $\Delta$ of diameter 0 and 1 are precisely the points and lines of $\Delta$. Convex subspaces of diameter 2,3 , respectively $n-1$, are called quads, hexes, respectively maxes. If $x$ is a point and $S$ is a convex subspace of $\Delta$, then $\pi_{S}(x)$ denotes the unique point of $S$ nearest to $x$. The point $\pi_{S}(x)$ is called the projection of $x$ onto $S$.

We will denote a dual polar space by putting a "D" in front of the name of the corresponding polar space. So, $D Q(2 n, q), D Q^{-}(2 n+1, q), D H\left(2 n-1, q^{2}\right)$, respectively $D W(2 n-1, q)$, denotes the dual polar space associated with a nonsingular quadric in $\mathrm{PG}(2 n, q)$, a nonsingular elliptic quadric in $\mathrm{PG}(2 n+1, q)$, a nonsingular hermitian variety in $\mathrm{PG}\left(2 n-1, q^{2}\right)$, respectively a symplectic polarity of $\operatorname{PG}(2 n-1, q)$.

\subsection{Embeddings}

In this paper, we will only consider embeddings in a finite-dimensional projective space. Let $\Delta$ be a thick dual polar space of rank at least 2 and let $V$ denote a finitedimensional vector space.

A projective embedding of $\Delta$ in $\Sigma=\mathrm{PG}(V)$ is an injective mapping $e$ from the point-set $P$ of $\Delta$ to the point-set of $\Sigma$ such that:

(E1) the image $e(P)$ of $e$ spans $\Sigma$;

(E2) every line of $\Delta$ is mapped by $e$ into a line of $\Sigma$;

(E3) no two lines of $\Delta$ are mapped by $e$ into the same line of $\Sigma$.

We will say that $e$ is an $\mathbb{F}$-embedding if $\mathbb{F}$ is the underlying division ring of the vector space $V$. The $\operatorname{dimensions} \operatorname{dim}(V)$ and $\operatorname{dim}(\Sigma)=\operatorname{dim}(V)-1$ are called the vector and projective dimension of $e$, respectively. Note that (E2) only says that the image $e(L)$ of a line $L$ of $\Delta$ is contained in a line of $\Sigma$. If $e(L)$ is a line of $\Sigma$ for every line $L$ of $\Delta$, then the embedding $e$ is said to be full. (Notice that in the literature, 
projective embeddings are often presupposed to be full.) For every point $p$ of $\Delta$, the hyperplane $H_{p}$ is a maximal subspace of $\Delta$. Hence, $e\left(H_{p}\right)$ spans either a hyperplane of $\Sigma$ or the whole of $\Sigma$. Following Thas and Van Maldeghem [16], we say that $e$ is polarized if $\left\langle e\left(H_{p}\right)\right\rangle$ is a hyperplane of $\Sigma$ for every point $p$ of $\Delta$. If this is the case, then $\left\langle e\left(H_{p}\right)\right\rangle \cap e(P)=e\left(H_{p}\right)$ (recall that $H_{p}$ is a maximal subspace). As noticed in [8, Remarks 2 and 3], if $n=\operatorname{rank}(\Delta)$ is sufficiently large (in any case, $n>2$ ), then $\Delta$ admits non-polarized full embeddings.

Given a projective embedding $e: \Delta \rightarrow \Sigma$, suppose that $\alpha$ is a subspace of $\Sigma$ satisfying the following properties:

(P1) $\alpha \cap e(P)=\emptyset$;

(P2) $\langle\alpha, e(x)\rangle \neq\langle\alpha, e(y)\rangle$ for every two distinct points $x$ and $y$ of $\Delta$.

For every point $x$ of $\Delta$, we define $e_{\alpha}(x):=\langle\alpha, e(x)\rangle$. Then $e_{\alpha}$ is an embedding of $\Delta$ in $\Sigma / \alpha$. We call $e_{\alpha}$ a projection of $e$. Both claims of the next lemma are obvious:

Lemma 1.1. If e is full, then also $e_{\alpha}$ is full. If $e_{\alpha}$ is polarized, then e is polarized.

Two embeddings $e_{1}: \Delta \rightarrow \Sigma_{1}$ and $e_{2}: \Delta \rightarrow \Sigma_{2}$ are called isomorphic $\left(e_{1} \cong e_{2}\right)$ if there exists an isomorphism $\phi$ from $\Sigma_{1}$ to $\Sigma_{2}$ such that $e_{2}(x)=\phi \circ e_{1}(x)$ for every point $x$ of $\Delta$.

Suppose $\tilde{\widetilde{V}}$ is a vector space over the division ring $\mathbb{F}$. Following Cooperstein and Shult [7] we say that a full $\mathbb{F}$-embedding $\tilde{e}: \Delta \rightarrow \widetilde{\Sigma}=\operatorname{PG}(\tilde{V})$ is absolutely universal (absolute for short) if for every full $\mathbb{F}$-embedding $e$ of $\Delta$, there exists a subspace $\alpha$ in $\widetilde{\Sigma}$ such that

(i) $\alpha$ satisfies properties (P1) and (P2) with respect to $\tilde{e}$ and $\tilde{e}_{\alpha} \cong e$.

The absolute embedding $\tilde{e}$, if it exists, is uniquely determined up to isomorphisms and satisfies the following, where $\alpha$ is as in (i):

(ii) for every full $\mathbb{F}$-embedding $e^{\prime}$ having a projection isomorphic to $e$, there exists a subspace $\alpha^{\prime}$ of $\alpha$ such that $\tilde{e}_{\alpha^{\prime}} \cong e^{\prime}$.

According to the terminology of Cooperstein and Shult [7] (see also Ronan [13]), (ii) just says that $\tilde{e}$ is universal relative to every full $\mathbb{F}$-embedding of $\Delta$. In other words, $\tilde{e}$ is the linear hull of every full $\mathbb{F}$-embedding of $\Delta$ (Pasini [12]). Sufficient conditions for a point-line geometry to admit the absolute full $\mathbb{F}$-embedding have been obtained by Kasikova and Shult [11].

If $e^{\prime}: Q \rightarrow \Sigma^{\prime}$ is a full embedding of a thick generalized quadrangle, then by Tits $[17,8.6]$, the underlying division ring of $\Sigma^{\prime}$ is completely determined by $Q$. Hence, if $e: \Delta \rightarrow \Sigma$ is a full embedding of a thick dual polar space of rank $n \geq 2$, then the underlying division ring of $\Sigma$ is completely determined by $\Delta$ (since $e$ induces a full embedding of each of its quads). This allows us to talk about full embeddings and absolutely universal embeddings of thick dual polar spaces, without mentioning the underlying division rings. 
The next proposition follows from Buekenhout and Lefèvre [4], Dienst [9] in the case of generalized quadrangles and from Kasikova and Shult [11, Section 4.6] in the case of thick dual polar spaces of rank at least 3.

Proposition 1.2. Every thick dual polar space of rank $n \geq 2$ admits the absolutely universal embedding, provided that it admits at least one full embedding.

The next proposition immediately follows from the second claim of Lemma 1.1:

Proposition 1.3. If a thick dual polar space admits a full polarized embedding, then its absolutely universal embedding is polarized.

\subsection{The main results of this paper}

Let $\Delta$ be a thick dual polar space of rank $n \geq 2$ admitting a full polarized embedding and let $P$ denote the point-set of $\Delta$. We will show the following in Section 2.

Theorem 1.4. Up to isomorphisms, there exists a unique full polarized embedding $\bar{e}$ such that every full polarized embedding e of $\Delta$ has a projection isomorphic to $\bar{e}$.

Let $\tilde{e}$ denote the absolutely universal full embedding of $\Delta$ (which exists by Proposition 1.2). With every full polarized embedding of $\Delta$, there are associated two projections:

$$
\tilde{e} \rightarrow e \rightarrow \bar{e},
$$

The embedding $\bar{e}$ is called the minimal full polarized embedding of $\Delta$.

\section{Definition}

(1) For every full embedding $e: \Delta \rightarrow \Sigma$ of $\Delta$ and for every convex subspace $F$ of $\Delta$, put $\Sigma_{F}:=\langle e(F)\rangle$ and let $e_{F}: F \rightarrow \Sigma_{F}$ denote the full embedding of $F$ induced by $e$.

(2) For every full polarized embedding $e: \Delta \rightarrow \Sigma$ of $\Delta$ and for every point $x$ of $\Delta$, let $e^{*}(x)$ denote the unique hyperplane of $\Sigma$ containing $e\left(H_{x}\right)$.

In Section 2, we will also prove the following theorems.

Theorem 1.5. If $e$ is a full polarized embedding of $\Delta$, then the embedding $e_{F}$ is polarized for every convex subspace $F$ of $\Delta$.

Theorem 1.6. Let $e: \Delta \rightarrow \Sigma$ be a full polarized embedding of $\Delta$ isomorphic to the minimal embedding of $\Delta$. Then for every convex subspace $F$ of $\Delta, e_{F}$ is isomorphic to the minimal full polarized embedding of $F$.

Now, suppose $e: \Delta \rightarrow \Sigma$ is a full polarized embedding of $\Delta$. For every point $x$ of $\Delta$, the unique hyperplane $e^{*}(x)$ of $\Sigma$ containing $e\left(H_{x}\right)$ is a point of $\Sigma^{*}$, the dual projective space of $\Sigma$. Let $\Sigma^{(*)}$ denote the subspace of $\Sigma^{*}$ generated by all points $e^{*}(x), x \in P$. We will show the following in Section 3: 
Theorem 1.7. $e^{*}: \Delta \rightarrow \Sigma^{(*)}$ is a full polarized embedding of $\Delta$.

We will call $e^{*}$ the dual embedding of $e$. In Section 3, we also show the following:

Theorem 1.8. The embedding $e^{*}$ is isomorphic to the minimal full polarized embedding of $\Delta$.

In the last section (Section 4), we will determine the dimension of the minimal full polarized embeddings for the following classes of dual polar spaces: $D W(2 n-1, q)$ (for $n \leq 5), D Q(2 n, q), D Q^{-}(2 n+1, q)$ and $D H\left(2 n-1, q^{2}\right)$.

\section{Minimal embeddings}

Let $\Delta$ be a thick dual polar space of rank $n \geq 2$ admitting a full polarized embedding and let $P$ denote the point-set of $\Delta$.

Definition If $e: \Delta \rightarrow \Sigma$ is a full polarized embedding of $\Delta$, then we define

$$
R_{e}:=\bigcap_{p \in P}\left\langle e\left(H_{p}\right)\right\rangle .
$$

Lemma 2.1. If $e: \Delta \rightarrow \Sigma$ is a full polarized embedding of $\Delta$ and if $\alpha \subseteq R_{e}$, then $\alpha$ satisfies the properties $(\mathrm{P} 1)$ and $(\mathrm{P} 2)$ of Section 1.2 and the embedding $e_{\alpha}: \Delta \rightarrow \Sigma / \alpha$ is polarized. In particular, this holds if $\alpha=R_{e}$.

Proof: We check property (P1). We have $R_{e} \cap e(P)=\bigcap_{p \in P}\left(\left\langle e\left(H_{p}\right)\right\rangle \cap e(P)\right)=$ $\bigcap_{p \in P} e\left(H_{p}\right)=e\left(\bigcap_{p \in P} H_{p}\right)=e(\emptyset)=\emptyset$. Hence, also $\alpha \cap e(P)=\emptyset$.

We check property (P2). Suppose that there exist distinct points $x$ and $y$ such that $\langle\alpha, e(x)\rangle=\langle\alpha, e(y)\rangle$. Take a point $z$ opposite $x$ such that $y$ lies on a shortest path between $x$ and $z$. (Such a point $z$ exists, see e.g. [2] where this property has been shown for a more general class of near polygons.) Since $y \in H_{z}$, the hyperplane $\left\langle e\left(H_{z}\right)\right\rangle$ of $\Sigma$ contains the point $e(y)$ and hence also the subspace $\langle\alpha, e(y)\rangle=\langle\alpha, e(x)\rangle$. So, $e(x) \in\left\langle e\left(H_{z}\right)\right\rangle \cap e(P)=e\left(H_{z}\right)$, contradicting $x \notin H_{z}$.

We will show that $e_{\alpha}$ is polarized. Let $p$ be any point of $\Delta$. Since $e$ is polarized, there exists a hyperplane $\Pi_{p}$ in $\Sigma$ through $\alpha \subseteq R_{e}$ containing all points of $e\left(H_{p}\right)$. It follows that the hyperplane $\Pi_{p} / \alpha$ of $\Sigma / \alpha$ contains all points of $e_{\alpha}\left(H_{p}\right)$.

Also the converse of Lemma 2.1 holds.

Lemma 2.2. Let e $: \Delta \rightarrow \Sigma$ be a full polarized embedding of $\Delta$ and let $\alpha$ be a subspace of $\Sigma$ satisfying properties $(\mathrm{P} 1)$ and $(\mathrm{P} 2)$ of Section 1.2. If $e_{\alpha}$ is polarized, then $\alpha \subseteq R_{e}$.

Proof: Suppose that $\alpha$ is not contained in $R_{e}$. Then there exists a point $x$ in $\Delta$ such that $\alpha \nsubseteq\left\langle e\left(H_{x}\right)\right\rangle$. But then $\left\langle e_{\alpha}\left(H_{x}\right)\right\rangle=\Sigma / \alpha$, contradicting the fact that $e_{\alpha}$ is polarized. 
Now, let $\tilde{e}: \Delta \rightarrow \widetilde{\Sigma}$ denote the absolutely universal embedding of $\Delta$. Then $\tilde{e}$ is polarized by Lemma 1.1 . We define

$$
\bar{e}:=\tilde{e}_{R_{\tilde{e}}} .
$$

We will now prove Theorem 1.4. Let $e$ be an arbitrary full polarized embedding of $\Delta$ and let $\alpha$ denote a subspace of $\widetilde{\Sigma}$ such that $\tilde{e}_{\alpha} \cong e$. By Lemma $2.2, \alpha \subseteq R_{\tilde{e}}$. Since $\alpha \subseteq R_{\tilde{e}}, e \cong \tilde{e}_{\alpha}$ must have a projection isomorphic to $\bar{e}=\tilde{e}_{R_{\tilde{e}}}$. The uniqueness of $\bar{e}$ is easy to see. If $\bar{e}_{1}$ and $\bar{e}_{2}$ are two embeddings satisfying the conditions of Theorem 1.4, then $\bar{e}_{1}$ is isomorphic to a projection of $\bar{e}_{2}$ and $\bar{e}_{2}$ is isomorphic to a projection of $\bar{e}_{1}$. This is only possible when $\bar{e}_{1} \cong \bar{e}_{2}$.

Remark 1. If $e: \Delta \rightarrow \Sigma$ is a full polarized embedding, then from Lemma 2.2 and Theorem 1.4, it readily follows that $e_{R_{e}}$ is isomorphic to the minimal full polarized embedding of $\Delta$.

We now prove Theorems 1.5 and 1.6.

Proof of Theorem 1.5. Let $\delta$ denote the diameter of $F$. Let $x$ denote an arbitrary point of $F$ and let $y$ denote a point of $\Delta$ at distance $n-\delta$ from $F$ such that $\Delta_{n-\delta}(y) \cap F=\{x\}$. So, for every point $z$ of $F, \mathrm{~d}(y, z)=\mathrm{d}(y, x)+\mathrm{d}(x, z)=n-\delta+\mathrm{d}(x, z)$. The points of $F$ contained in $H_{y}$ are precisely the points of $F$ contained in $\Delta_{\delta-1}^{*}(x)$, where $\Delta_{\delta-1}^{*}(x)$ stands for the set of points of $\Delta$ at distance at most $\delta-1$ from $x$. This implies that (i) $\left\langle e\left(H_{y}\right)\right\rangle \cap \Sigma_{F}$ is a hyperplane of $\Sigma_{F}$, and (ii) $e_{F}\left(\Delta_{\delta-1}^{*}(x) \cap F\right) \subseteq\left\langle e\left(H_{y}\right)\right\rangle \cap \Sigma_{F}$. It follows that $\left\langle e_{F}\left(\Delta_{\delta-1}^{*}(x) \cap F\right)\right\rangle$ coincides with the hyperplane $\left\langle e\left(H_{y}\right)\right\rangle \cap \Sigma_{F}$ of $\Sigma_{F}$. Hence, $e_{F}$ is polarized.

Proof of Theorem 1.6: By Theorem 1.5, $e_{F}$ is polarized. We must still show that $R_{e_{F}}=\emptyset$ :

$$
\begin{aligned}
R_{e_{F}} & =\bigcap_{x \in F} e_{F}^{*}(x)=\bigcap_{x \in \Delta_{n-\delta}(F)}\left(e^{*}(x) \cap \Sigma_{F}\right)=\bigcap_{x \in P}\left(e^{*}(x) \cap \Sigma_{F}\right) \\
& =\left(\bigcap_{x \in P} e^{*}(x)\right) \cap \Sigma_{F}=\emptyset \cap \Sigma_{F}=\emptyset .
\end{aligned}
$$

\section{Dual embeddings}

Again, let $\Delta$ be a thick dual polar space of rank $n \geq 2$. Notice that two lines $L$ and $M$ of $\Delta$ are at maximal distance from each other if and only if $\mathrm{d}(L, M)=n-1$.

Lemma 3.1. Let $a$ and $b$ be distinct points of a line $L$ of $\Delta$, and let $H$ be a hyperplane of $\Delta$ containing $H_{a} \cap H_{b}$. Then either $H=H_{c}$ for a (unique) point $c \in L$ or $H$ contains a line $L^{\prime}$ with $\mathrm{d}\left(L, L^{\prime}\right)=n-1$. 
Proof: Notice first that, since $\Delta$ is a near polygon, $H_{a} \cap H_{b}$ consists of those points of $\Delta$ that have distance at most $n-2$ from $L$. If $H$ contains a line $L^{\prime}$ with $\mathrm{d}\left(L, L^{\prime}\right)=$ $n-1$, then we are done. So, suppose that $|M \cap H|=1$ for every line $M$ of $\Delta$ with $\mathrm{d}(M, L)=n-1$. For every such line $M$, we denote by $c(M)$ the unique point of $L$ at distance $n-1$ from the point $M \cap H$.

\section{Claim 1. Suppose $L_{1}$ and $L_{2}$ are two lines of $\Delta$ satisfying}

(i) $L_{1}$ and $L_{2}$ are contained in a quad $Q$,

(ii) $\mathrm{d}\left(L_{1}, L\right)=d\left(L_{2}, L\right)=n-1$.

Then $c\left(L_{1}\right)=c\left(L_{2}\right)$.

Since $\mathrm{d}\left(L_{1}, L\right)=n-1$, every point of $L$ has distance at least $n-2$ (and hence precisely $n-2$ ) from $Q$. Put $L^{\prime}:=\pi_{Q}(L)$. Then $L^{\prime}$ is a line. (If $\pi_{Q}(L)$ is a point, then not every point of $L$ would have the same distance from $Q$.) All points of $L^{\prime}$ have distance $n-2$ from $L$. So, $H$ contains $L^{\prime}, p_{1}$ and $p_{2}$, where $p_{i}, i \in\{1,2\}$, is the unique point of $L_{i}$ contained in $H$. Let $q_{i}$ denote the unique point of $L^{\prime}$ collinear with $p_{i}$. Then, $q_{i}$ is the unique point of $Q$ at distance $n-2$ from $c_{i}:=c\left(L_{i}\right)$. Conversely, $c_{i}$ is the unique point of $L$ at distance $n-2$ from $q_{i}$. So, if $q_{1}=q_{2}$ then $c_{1}=c_{2}$ and we are done. By way of contradiction, assume that $q_{1} \neq q_{2}$. Then $Q \cap H$ is a nondegenerate subquadrangle of $Q$, since $Q \cap H$ contains the two disjoint lines $p_{1} q_{1}$ and $p_{2} q_{2}$. In that subquadrangle we can find a line $M$ with $M \cap L^{\prime}=\emptyset$. Clearly, $\mathrm{d}\left(M, L^{\prime}\right)=1$, whence $\mathrm{d}(M, L)=n-1$. However, $M \subset H$, contrary to the assumption that $|M \cap H|=1$ for every line $M$ with $\mathrm{d}(M, L)=n-1$. Therefore $q_{1}=q_{2}$, as we wanted to prove.

Claim 2. The point $c=c(M)$ does not depend on the choice of the line $M$ at distance $n-1$ from $L$.

Indeed, the lines at distance $n-1$ from $L$ are the lines of the geometry $\operatorname{Far}_{\Delta}(L)$ formed by the elements of $\Delta$ at maximal distance from $L$, with the incidence relation inherited from $\Delta$. This geometry is residually connected (Blok and Brouwer [1]). So, the graph having the lines far from $L$ as vertices and 'being in the same quad' as the adjacency relation is connected. Claim 2 follows from this and Claim 1.

We can now finish the proof of the lemma. Put $c:=c(M)$ for $M$ a line with $\mathrm{d}(M, L)=$ $n-1$. In view of Claim 2, $c$ does not depend on the choice of the line $M$. Let $x \in H_{c}$. If $\mathrm{d}(x, L) \leq n-2$, then $x \in H$ since $x \in H_{a} \cap H_{b}$. If $\mathrm{d}(x, L)=n-1$, let $X$ be a line through $x$ not contained in the unique max through $x$ and $c$. Then $\mathrm{d}(X, L)=n-1$ and $\mathrm{d}(c, X \cap H)=n-1$, by Claim 2. On the other hand, $X \cap \Delta_{n-1}(c)=\{x\}$, by the choice of $X$. Therefore $\{x\}=X \cap H$. Hence $\Delta_{n-1}(L) \cap \Delta_{n-1}(c) \subseteq H$. It follows that $H_{c} \subseteq H$ and hence $H_{c}=H$ since $H_{c}$ is a maximal subspace.

Henceforth, $e: \Delta \rightarrow \Sigma=P G(V)$ is a given full polarized embedding of $\Delta$. We denote by $P$ the point-set of $\Delta$ and, for a subset $X \subseteq P$, we put:

$$
\langle X\rangle_{e}:=\langle e(X)\rangle_{\Sigma}
$$


(The subscript $\Sigma$ in the symbol $\langle\cdot\rangle_{\Sigma}$ should remind us of the space $\Sigma$ where spans are taken.) Since $e$ is polarized,

(P) $\left\langle H_{p}\right\rangle_{e} \neq \Sigma$, for every point $p$ of $\Delta$.

By Shult [14, Lemma 6.1], every hyperplane of $\Delta$ is maximal as a subspace of $\Delta$. Therefore, if $H$ is a hyperplane of $\Delta$ and $\langle H\rangle_{e} \neq \Sigma$, then $\langle H\rangle_{e}$ is a hyperplane of $\Sigma$ (recall also property (E1)) and $\langle H\rangle_{e} \cap e(P)=e(H)$. Accordingly, if $H_{1}, H_{2}$ are different hyperplanes of $\Delta$ with $\left\langle H_{i}\right\rangle_{e} \neq \Sigma$ for $i=1,2$, then $\left\langle H_{1}\right\rangle_{e} \cap\left\langle H_{2}\right\rangle_{e}$ has codimension 2 in $\Delta$. These facts will be freely used in the sequel.

Lemma 3.2. Let $H$ be a hyperplane of $\Delta$ and let $L$ be a line of $\Delta$ containing two points $a$ and $b$ such that $\left\langle H_{a}\right\rangle_{e} \cap\left\langle H_{b}\right\rangle_{e} \subseteq\langle H\rangle_{e} \neq \Sigma$. Then $H=H_{c}$ for some point $c \in L$.

Proof: Since $\left\langle H_{a}\right\rangle_{e} \cap\left\langle H_{b}\right\rangle_{e} \subseteq\langle H\rangle_{e}, \quad\left(\left\langle H_{a}\right\rangle_{e} \cap e(P)\right) \cap\left(\left\langle H_{b}\right\rangle_{e} \cap e(P)\right) \subseteq\left(\langle H\rangle_{e} \cap\right.$ $e(P))$ or $e\left(H_{a}\right) \cap e\left(H_{b}\right) \subseteq e(H)$. Hence, $H_{a} \cap H_{b} \subseteq H$. If $H$ contains a line $L^{\prime}$ at distance $n-1$ from $L$, then $H$ contains a point $p \in L^{\prime} \cap \Delta_{n-1}(a)$. Note that $\mathrm{d}(p, b)=n$, as $\mathrm{d}\left(L^{\prime}, L\right)=n-1$. Turning to spans in $\Sigma,\langle H\rangle_{e}$ contains $e(p)$ and the subspace $S=\left\langle H_{a}\right\rangle_{e} \cap\left\langle H_{b}\right\rangle_{e}$ of $S$. However, $S$ has codimension 2 in $\Sigma$ and does not contain $e(p)$, whereas $S \cup\{e(p)\}$ spans $\left\langle H_{a}\right\rangle_{e}$. It follows that $\langle H\rangle_{e}=\left\langle H_{a}\right\rangle_{e}$. Similarly, $\langle H\rangle_{e}=\left\langle H_{b}\right\rangle_{e}$. Hence $\left\langle H_{a}\right\rangle_{e}=\left\langle H_{b}\right\rangle_{e}$, which is impossible. Therefore, $H$ does not contain any line at distance $n-1$ from $L$. By Lemma 3.1, $H=H_{c}$ for some point $c \in L$.

Lemma 3.3. Let $a, b$ and $c$ be three distinct points of a line $L$ of $\Delta$. Then $\left\langle H_{c}\right\rangle_{e} \supseteq$ $\left\langle H_{a}\right\rangle_{e} \cap\left\langle H_{b}\right\rangle_{e}$.

Proof: Let $x$ be a point of $\Delta_{n-1}(c) \cap \Delta_{n}(a) \cap \Delta_{n}(b)$. Then $x \notin H_{a} \cup H_{b}$. So, $e(x) \notin\left\langle H_{a}\right\rangle_{e} \cup\left\langle H_{b}\right\rangle_{e}$ and $\Pi=\left\langle e(x),\left\langle H_{a}\right\rangle_{e} \cap\left\langle H_{b}\right\rangle_{e}\right\rangle$ is a hyperplane of $\Sigma$. Put $H:=e^{-1}(\Pi \cap e(P))$. Then $\left\langle H_{a}\right\rangle_{e} \cap\left\langle H_{b}\right\rangle_{e} \subseteq\langle H\rangle_{e}=\Pi \neq \Sigma$. By Lemma 3.2, $H=$ $H_{c^{\prime}}$ for some point $c^{\prime}$ of $a b$. Since $x \in H_{c^{\prime}}, \mathrm{d}\left(x, c^{\prime}\right) \leq n-1$. Hence, $c^{\prime}=c$ and $\left\langle H_{a}\right\rangle_{e} \cap\left\langle H_{b}\right\rangle_{e} \subseteq\left\langle H_{c}\right\rangle_{e}$.

Put $e^{*}(p):=\left\langle H_{p}\right\rangle_{e}$ for every point $p \in P$. Note that, as $e$ is polarized, $e^{*}(p)$ is indeed a point of $\Sigma^{*}$. Since $e^{*}(p) \cap e(P)=e\left(H_{p}\right)$ for every point $p$ of $\Delta, e^{*}$ is injective. Moreover, $e^{*}$ maps lines of $\Delta$ onto lines of $\Sigma^{*}$, by Lemmas 3.2 and 3.3. Therefore, $e^{*}$ is an embedding of $\Delta$ in $\Sigma^{(*)}$, where we denote by $\Sigma^{(*)}$ the span of $e^{*}(P)$ in $\Sigma^{*}$ :

$$
\Sigma^{(*)}:=\left\langle e^{*}(P)\right\rangle_{\Sigma^{*}}
$$

Note that

$$
\Sigma^{(*)} \cong\left(\Sigma / R_{e}\right)^{*},
$$


where $\left(\Sigma / R_{e}\right)^{*}$ is the dual of $\Sigma / R_{e}$. The following lemma finishes the proof of Theorem 1.7.

\section{Lemma 3.4. $e^{*}$ is polarized.}

Proof: Let $p$ denote an arbitrary point of $\Delta$. We must show that there exists a hyperplane in $\Sigma^{(*)}$ containing all points $e^{*}(x)$ with $x \in H_{p}$. If $x \in H_{p}$, then $p \in H_{x}$ and hence $e(p) \in e^{*}(x)$. Hence, the hyperplane $\left\langle R_{e}, e(p)\right\rangle$ of $\Sigma^{(*)}$ contains all points $e^{*}(x)$, $x \in H_{p}$. This proves the lemma.

Theorem 1.8 now also readily follows. Since the minimal full polarized embedding $\bar{e}$ of $\Delta$ is isomorphic to $e_{R_{e}}$, its projective dimension is equal to $\operatorname{dim}(\Sigma)-\operatorname{dim}\left(R_{e}\right)-1$. On the other hand, since $\Sigma^{(*)} \cong\left(\Sigma / R_{e}\right)^{*}, e^{*}$ has also projective dimension $\operatorname{dim}(\Sigma)-$ $\operatorname{dim}\left(R_{e}\right)-1$, Hence, $\bar{e}$ and $e^{*}$ are isomorphic embeddings.

\section{Examples}

Although we believe that the treatments given below might also hold for several infinite fields, we will only consider the finite case, for which we can rely on some published material.

In Sections 4.1 and 4.2, we will show that the natural embeddings of the dual polar spaces $D Q(2 n, q), D Q^{-}(2 n+1, q)$ and $D H\left(2 n-1, q^{2}\right)$ are also their minimal ones. In Section 4.3, it will be shown that this is not always the case for the dual polar sace $D W(2 n-1, q)$.

Remark 2. One of the referees pointed out to the authors that the fact that the natural embeddings of $D Q(2 n, q), D Q^{-}(2 n+1, q)$ and $D H\left(2 n-1, q^{2}\right)$ are minimal also follows from the irreducibility of the associated modules.

Let $\Delta$ be one of the dual polar spaces $D Q(2 n, q), D Q^{-}(2 n+1, q)$ or $D H(2 n-$ $1, q^{2}$ ), and let $e: \Delta \rightarrow \Sigma$ denote the natural embedding of $\Delta$ (see Sections 4.1 and 4.2). Then $G=\operatorname{Aut}(\Delta)$ lifts to a group $\widetilde{G}$ of automorphisms of $\Sigma$. In each of the three cases, it can be shown that the module $(\Sigma, \widetilde{G})$ is irreducible. As each element of $\widetilde{G}$ fixes $R_{e}$, we necessarily must have that $R_{e}=\emptyset$, i.e., $e$ is isomorphic to the minimal full polarized embedding of $\Delta$. However, the arguments we are going to exploit in Sections 4.1 and 4.2 are far more straightforward than the above.

\subsection{Minimal embeddings of $D Q(2 n, q)$ and $D Q^{-}(2 n+1, q)$}

By Corollary 1.5 of De Bruyn and Pasini [8], every polarized embedding of a dual polar space of rank $n$ has vector dimension at least $2^{n}$.

The dual polar space $D Q(2 n, q)$ admits a polarized full embedding $e_{\text {spin }}$ of vector dimension $2^{n}$, called the spin-embedding. We refer to Buekenhout and Cameron [3] for a description of $e_{\text {spin }}$. If $q$ is odd, then the embedding $e_{\text {spin }}$ is absolutely universal (Wells [18]; see also Cooperstein and Shult [7]) and hence is the unique polarized 
full embedding of $D Q(2 n, q)$. If $q$ is even, then $e_{\text {spin }}$ is the minimal polarized full embedding of $D Q(2 n, q)$.

The dual polar space $D Q^{-}(2 n+1, q)$ admits a polarized full embedding $e_{\text {spin }}^{-}$induced by the natural embedding of the half-spin geometry of $Q^{+}\left(2 n+1, q^{2}\right)$, see Cooperstein and Shult [7]. The embedding $e_{\text {spin }}^{-}$is absolutely universal, no matter if $q$ is odd or even, and hence is the unique polarized full embedding of $D Q^{-}(2 n+1, q)$.

4.2. Minimal polarized full embeddings of $D H\left(2 n-1, q^{2}\right)$

Let $H\left(2 n-1, q^{2}\right)$ denote a non-singular hermitian variety in $\operatorname{PG}\left(2 n-1, q^{2}\right)$, put $N:=\left(\begin{array}{c}2 n \\ n\end{array}\right)$ and $I:=\{1, \ldots, 2 n\}$. For every subset $J$ of $I$, we define $\sigma(J)=(1+$ $\cdots+|J|)+\Sigma_{j \in J} j$.

Suppose $X$ is an $(n-1)$-dimensional subspace of $\operatorname{PG}\left(2 n-1, q^{2}\right)$ generated by the points $\left(x_{i, 1}, \ldots, x_{i, 2 n}\right), 1 \leq i \leq n$, of $\mathrm{PG}\left(2 n-1, q^{2}\right)$. For every $J=\left\{i_{1}, i_{2}, \ldots, i_{n}\right\}$ in $\left(\begin{array}{l}I \\ n\end{array}\right)$ with $i_{1}<i_{2}<\cdots<i_{n}$, we define

$$
X_{J}:=\left|\begin{array}{cccc}
x_{1, i_{1}} & x_{1, i_{2}} & \cdots & x_{1, i_{n}} \\
x_{2, i_{1}} & x_{2, i_{2}} & \cdots & x_{2, i_{n}} \\
\vdots & \vdots & \ddots & \vdots \\
x_{n, i_{1}} & x_{n, i_{2}} & \cdots & x_{n, i_{n}}
\end{array}\right| .
$$

The elements $X_{J}, J \in\left(\begin{array}{l}I \\ n\end{array}\right)$, are the coordinates of a point $f(X)$ of $\operatorname{PG}\left(N-1, q^{2}\right)$ and this point does not depend on the particular set of $n$ points which we have chosen as generating set for $X$. The elements $X_{J}, J \in\left(\begin{array}{l}I \\ n\end{array}\right)$, are called the Grassmann coordinates of $X$. (For more details on this topic, we refer to [10, Chapter 24]). By [5, Proposition 5.1], there exists a Baer subgeometry $\mathrm{PG}(N-1, q)$ of $\mathrm{PG}\left(N-1, q^{2}\right)$ containing all points $f(X)$ where $X$ is a generator (i.e. a maximal subspace) of $H\left(2 n-1, q^{2}\right)$. In this way, we obtain a map $e$ from the point set of $D H\left(2 n-1, q^{2}\right)$ to the point set of $\operatorname{PG}(N-1, q)$, which we like to call the Grassmann embedding of $D H\left(2 n-1, q^{2}\right)$. By [5], $e$ is indeed a full embedding. Moreover, $e$ is absolutely universal if $q \neq 2$.

Now, let $X$ and $Y$ be two generators of $H\left(2 n-1, q^{2}\right)$. Suppose $X$ is generated by the points $\left(x_{i, 1}, \ldots, x_{i, 2 n}\right), 1 \leq i \leq n$, and that $Y$ is generated by the points $\left(y_{i, 1}, \ldots, y_{i, 2 n}\right)$, $1 \leq i \leq n$. Obviously, the points $X$ and $Y$ are at non-maximal distance if and only if

$$
\left|\begin{array}{llll}
x_{1,1} & x_{1,2} & \cdots & x_{1,2 n} \\
\vdots & \vdots & \ddots & \vdots \\
x_{n, 1} & x_{n, 2} & \cdots & x_{n, 2 n} \\
y_{1,1} & y_{1,2} & \cdots & y_{1,2 n} \\
\vdots & \vdots & \ddots & \vdots \\
y_{n, 1} & y_{n, 2} & \cdots & y_{n, 2 n}
\end{array}\right|=0
$$


i.e., if and only if

$$
\sum_{J \in\left(\begin{array}{l}
l \\
n
\end{array}\right)}(-1)^{\sigma(J)} X_{J} Y_{I \backslash J}=0
$$

(Expand according to the first $n$ rows.) For a given point $X$ of $D H\left(2 n-1, q^{2}\right)$, Eq. (1) defines a hyperplane of $\operatorname{PG}(N-1, q)$. This implies that $e$ is a polarized embedding. We will now show that $R_{e}=\emptyset$. To that goal, consider $N$ points $X_{1}, \ldots, X_{N}$ in $D H\left(2 n-1, q^{2}\right)$ such that $e\left(X_{1}\right), \ldots, e\left(X_{N}\right)$ generate $\operatorname{PG}(N-1, q)$. (Such points exist by property (E1).) Since the points $e\left(X_{1}\right), \ldots, e\left(X_{N}\right)$ are also linearly independent, the hyperplanes $e^{*}\left(X_{1}\right), \ldots, e^{*}\left(X_{N}\right)$ are linearly independent by Eq. (1). This implies that $R_{e}=\emptyset$.

From the previous discussion, the following theorem readily follows.

Theorem 4.1. - The Grassmann embedding of $D H\left(2 n-1, q^{2}\right)$ is the minimal polarized full embedding of $\mathrm{DH}\left(2 n-1, q^{2}\right)$.

- If $q \neq 2$, then the Grassmann embedding is the unique (up to isomorphisms) polarized full embedding of $\mathrm{DH}\left(2 n-1, q^{2}\right)$.

\subsection{Minimal polarized full embeddings of $D W(2 n-1, q)$}

Let $\zeta$ be a symplectic polarity in $\operatorname{PG}(2 n-1, q)$. Let $W(2 n-1, q)$ and $D W(2 n-$ $1, q)$ denote the associated polar and dual polar spaces. Put $K:=\{1, \ldots, n\}, I:=$ $\{1, \ldots, 2 n\}$ and $N:=\left(\begin{array}{c}2 n \\ n\end{array}\right)-\left(\begin{array}{c}2 n \\ n-2\end{array}\right)$. For every $i \in I$, we define $i^{\prime}:=i+n$. For every subset $J$ of $I$, we define $\sigma(J)=(1+\cdots+|J|)+\sum_{j \in J} j$ and $J^{\prime}:=\left\{j^{\prime} \mid j \in J\right\}$.

Let $X$ be an $(n-1)$-dimensional subspace of $\operatorname{PG}(2 n-1, q)$. As in Section 4.2, let $X_{J}, J \in\left(\begin{array}{l}I \\ n\end{array}\right)$, denote the Grassmann coordinates of $X$. These coordinates define a point $f(X)=\left\langle\sum_{J} X_{J} e_{J}\right\rangle$ in $\left.\operatorname{PG}\left(\begin{array}{c}2 n \\ n\end{array}\right)-1, q\right)$. By [6, Proposition 5.1], the subspace

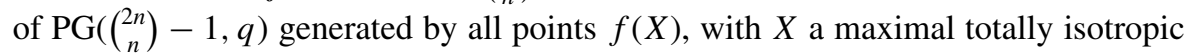
subspace of $W(2 n-1, q)$, is $(N-1)$-dimensional. We will denote this subspace by $\operatorname{PG}(N-1, q)$. So, we obtain a map $e$ from the point set of $D W(2 n-1, q)$ to the point set of $\operatorname{PG}(N-1, q)$. By [6], $e$ is an absolutely universal full embedding. We will call $e$ the Grassmann embedding of $D W(2 n-1, q)$. With a similar reasoning as in Section 4.2, we find that two points $X$ and $Y$ of $D W(2 n-1, q)$ are at non-maximal distance if and only if

$$
\sum_{J \in\left(\begin{array}{l}
l \\
n
\end{array}\right)}(-1)^{\sigma(J)} X_{J} Y_{I \backslash J}=0
$$

It follows again that $e$ is polarized. The Eq. (2) determines a bilinear form in the $N$-dimensional vector space $V(N, q)$ associated with $\operatorname{PG}(N-1, q)$. Since $\sigma(J)+$ $\sigma(I \backslash J) \equiv n \quad(\bmod 2)$ for $J \in\left(\begin{array}{l}I \\ n\end{array}\right)$, this bilinear form is symmetric if $n$ is even and alternating if $n$ is odd. The space $R_{e}$ corresponds with the radical of this bilinear form. So, the dimension of the minimal polarized full embedding of $D W(2 n-1, q)$ is equal to the rank of any $(N \times N)$-matrix $M$ which represents the form (2) with respect to a certain basis of $V(N, q)$. In the sequel, we will use the following convention to denote 
such a matrix $M$ : if $A_{i}, i \in\{1, \ldots, k\}$, is an $\left(n_{i} \times n_{i}\right)$-matrix, then $\operatorname{diag}\left(A_{1}, \ldots, A_{k}\right)$ denotes the $\left(n_{1}+\cdots+n_{k}\right) \times\left(n_{1}+\cdots+n_{k}\right)$-matrix

$$
\left[\begin{array}{cccc}
A_{1} & & & \\
& A_{2} & & \\
& & \ddots & \\
& & & A_{k}
\end{array}\right] \text {, }
$$

where all entries outside the blocks $A_{1}, A_{2}, \ldots, A_{k}$ are null. We will now calculate the rank of such a matrix $M$ in the case that $n \in\{3,4,5\}$. We omit the case $n \geq 6$, since some of the calculations to perform become too tiresome, and we have not discovered a way to speed them up in a general setting, suited for any $n$. Of course, it is also possible to do the calculations for $n=2$, but we will not do that since the polarized full embeddings of the generalized quadrangle $D W(3, q) \cong Q(4, q)$ are well-known, see [4].

Suppose that the symplectic polarity $\zeta$ defining $W(2 n-1, q)$ is represented by the following matrix:

$$
\left[\begin{array}{cc}
0_{n} & I_{n} \\
-I_{n} & 0_{n}
\end{array}\right]
$$

If $X$ is a totally isotropic $(n-1)$-space of $\mathrm{PG}(2 n-1, q)$ whose Grassmann coordinate $X_{K}$ is different from 0 , then there exists an $(n \times n)$-matrix $B$ such that $X$ is generated by the $n$ rows of $\left[I_{n} B\right]$. The fact that $X$ is totally isotropic then implies that $B=B^{T}$.

Lemma 4.2. Let $\Delta$ be a thick dual polar space of rank $n \geq 1$. (By convention, dual polar spaces of rank 1 are just lines.) If $H$ is a hyperplane of $\Delta$, then the smallest subspace $S$ of $\Delta$ containing $\Delta \backslash H$ coincides with $\Delta$.

Proof: We will prove this by induction on the rank $n$. Obviously, the lemma holds if $n=1$. So, suppose that $n \geq 2$ and that the lemma holds for any thick dual polar space of rank at most $n-1$. Let $x$ denote an arbitrary point of $\Delta$. If $M$ is a max through $x$ not contained in $H$, then $M \cap H$ is a hyperplane of $M$ and by the induction hypothesis applied to $M$, we then know that $x \in S$. Suppose therefore that every max through $x$ is contained in $H$. Then $H_{x} \subseteq H$ and hence $H_{x}=H$ since $H_{x}$ is a maximal subspace. By downwards induction on $i \in\{0, \ldots, n\}$, one easily proves that any point of $\Delta_{i}(x)$ belongs to $S$. In particular, we have that $x \in S$. This proves the lemma.

Corollary 4.3. Let $H$ be the hyperplane of $D W(2 n-1, q)$ which consists of all points of $D W(2 n-1, q)$ whose Grassmann coordinate $X_{K}$ is equal to 0 . Then any subspace of $D W(2 n-1, q)$ containing all points of $D W(2 n-1, q) \backslash H$ coincides with $D W(2 n-1, q)$.

Note that, by the remark preceding Lemma 4.2, points of $D W(2 n-1, q)$ with Grassmann coordinate $X_{K} \neq 0$ actually exist. 


\section{The rank 3 case}

In this case $e$ determines an embedding of $D W(5, q)$ into a subspace PG(13, $q)$ of $\operatorname{PG}(19, q)$.

Lemma 4.4. The subspace $\mathrm{PG}(13, q)$ of $\mathrm{PG}(19, q)$ is described by the following 6 equations: $X_{235}=-X_{134} ; X_{236}=X_{124} ; X_{136}=-X_{125} ; X_{346}=-X_{245} ; X_{356}=$ $X_{145} ; X_{256}=-X_{146}$.

Proof: In view of Corollary 4.3, it suffices to show that all points $X$ of $D W(5, q)$ with $X_{K} \neq 0$ satisfy these equations. Such a point $X$ is generated by the rows of a $(3 \times 6)$-matrix $\left[I_{3} B\right]$ with $B=B^{T}$, i.e., by the rows of a matrix of the following form:

$$
\left[\begin{array}{cccccc}
1 & 0 & 0 & b_{11} & b_{12} & b_{13} \\
0 & 1 & 0 & b_{12} & b_{22} & b_{23} \\
0 & 0 & 1 & b_{13} & b_{23} & b_{33}
\end{array}\right]
$$

One easily verifies that $X_{235}=b_{12}=-X_{134}, X_{236}=b_{13}=X_{124}, X_{136}=-b_{23}=$ $-X_{125}, \quad X_{346}=b_{11} b_{23}-b_{12} b_{13}=-X_{245}, \quad X_{356}=b_{12} b_{23}-b_{22} b_{13}=X_{145} \quad$ and $X_{256}=b_{13} b_{23}-b_{12} b_{33}=-X_{146}$.

By Lemma $4.4, \quad \mathcal{B}=\left(e_{123}, e_{456}, e_{345}, e_{126}, e_{246}, e_{135}, e_{156}, e_{234}, e_{356}+e_{145}\right.$, $\left.e_{124}+e_{236}, e_{346}-e_{245}, e_{136}-e_{125}, e_{256}-e_{146}, e_{235}-e_{134}\right)$ is an ordered basis of the vector space $V(14, q)$ associated with $\operatorname{PG}(13, q)$. With respect to this basis, the bilinear form (2) has matrix $\operatorname{diag}\left(M_{1}, M_{1}, M_{1}, M_{1}, M_{2}, M_{2}, M_{2}\right)$, with $M_{1}=\left(\begin{array}{rr}0 & 1 \\ -1 & 0\end{array}\right)$ and $M_{2}=\left(\begin{array}{rr}0 & 2 \\ -2 & 0\end{array}\right)$.

From the previous discussion, the following result readily follows.

Theorem 4.5. If $q \neq 2^{r}$, then the Grassmann embedding of $D W(5, q)$ is the unique polarized full embedding of $D W(5, q)$. If $q=2^{r}$, then $D W(5, q) \cong D Q(6, q)$ and the minimal full polarized embedding of $D W(5, q)$ is the spin embedding (of vector dimension 8).

\section{The rank 4 case}

In this case $e$ determines an embedding of $D W(7, q)$ into a subspace $\mathrm{PG}(41, q)$ of $\operatorname{PG}(69, q)$.

Lemma 4.6. The subspace $\mathrm{PG}(41, q)$ of $\mathrm{PG}(69, q)$ is described by the following 28 equations.

- For all subsets $H_{1}$ and $H_{2}$ of $K$ satisfying $\left|H_{1}\right|=\left|H_{2}\right|$ and $H_{1} \neq H_{2}$,

$$
(-1)^{\sigma\left(K \backslash H_{1}\right)} X_{\left(K \backslash H_{1}\right) \cup H_{2}^{\prime}}=(-1)^{\sigma\left(K \backslash H_{2}\right)} X_{\left(K \backslash H_{2}\right) \cup H_{1}^{\prime}} .
$$

- $X_{2367}+X_{2468}=-X_{3478}$. 
Proof: Similarly as in the proof of Lemma 4.4, we may suppose the point $X$ of $D W(2 n-1, q)$ is generated by the $n$ rows of the matrix $\left[I_{n} B\right]$, with $B=\left(b_{i j}\right)_{1 \leq i, j \leq n}$ such that $b_{i j}=b_{j i}$ for all $i, j \in\{1, \ldots, n\}$. Let $C$ denote the square submatrix of $B$ consisting of all entries $b_{i j}$ with $i \in H_{1}$ and $j \in H_{2}$. Let $C^{\prime}$ denote the square submatrix of $B$ consisting of all entries $b_{i j}$ with $i \in H_{2}$ and $j \in H_{1}$. Then $C^{\prime}=C^{T}$. Now,

$$
(-1)^{\sigma\left(K \backslash H_{1}\right)} X_{\left(K \backslash H_{1}\right) \cup H_{2}^{\prime}}=\operatorname{det}(C)=\operatorname{det}\left(C^{\prime}\right)=(-1)^{\sigma\left(K \backslash H_{2}\right)} X_{\left(K \backslash H_{2}\right) \cup H_{1}^{\prime}} .
$$

The equality $X_{2367}+X_{2468}=-X_{3478}$ follows from a direct verification.

Consider now the ordered basis $\mathcal{B}$ of $V(42, q)$ consisting of the following 16 vectors of weight 1 :

$e_{1234}, e_{5678}, e_{1238}, e_{4567}, e_{1247}, e_{3568}, e_{1278}, e_{3456}, e_{1346}, e_{2578}, e_{1467}, e_{2358}$,

$e_{1678}, e_{2345}, e_{2457}, e_{1368}$,

the following 24 vectors of weight 2 :

$$
\begin{aligned}
& e_{1235}-e_{2348}, e_{1567}-e_{4678}, e_{1236}+e_{1348}, e_{4578}+e_{2567}, \\
& e_{1237}-e_{1248}, e_{3567}-e_{4568}, e_{1245}+e_{2347}, e_{3678}+e_{1568}, \\
& e_{1246}-e_{1347}, e_{2568}-e_{3578}, e_{1257}-e_{2478}, e_{1356}-e_{3468}, \\
& e_{1258}+e_{2378}, e_{3467}+e_{1456}, e_{1267}+e_{1478}, e_{3458}+e_{2356}, \\
& e_{1268}-e_{1378}, e_{2456}-e_{3457}, e_{1345}-e_{2346}, e_{1578}-e_{2678}, \\
& e_{1358}-e_{2368}, e_{1457}-e_{2467}, e_{1367}-e_{1468}, e_{2357}-e_{2458},
\end{aligned}
$$

and the following 2 vectors of weight 4 :

$$
e_{1357}+e_{2468}-e_{1256}-e_{3478}, e_{1458}+e_{2367}-e_{1256}-e_{3478} .
$$

With respect to this basis, the bilinear form is represented by the matrix

$$
\operatorname{diag}(\underbrace{M_{3}, \ldots, M_{3}}_{8 \text { times }}, \underbrace{M_{4}, \ldots, M_{4}}_{12 \text { times }}, M_{5}),
$$

where $M_{3}=\left(\begin{array}{ll}0 & 1 \\ 1 & 0\end{array}\right), M_{4}=\left(\begin{array}{ll}0 & 2 \\ 2 & 0\end{array}\right)$ and $M_{5}=\left(\begin{array}{ll}4 & 2 \\ 2 & 4\end{array}\right)$. Note that $\operatorname{det}\left(M_{5}\right)=12$.

From the previous discussion, the following theorem readily follows.

Theorem 4.7. - If $q=2^{r}$, then $D W(7, q) \cong D Q(8, q)$ and the minimal polarized full embedding of $D W(7, q)$ is the spin embedding (vector dimension 16);

- If $q=3^{r}$, then the minimal polarized full embedding of $D W(7, q)$ has vector dimension 41;

- If $2^{r} \neq q \neq 3^{r}$, then the Grassmann embedding of $D W(7, q)$ (of vector dimension 42) is the unique polarized full embedding of $D W(7, q)$.

\section{The rank 5 case}

In this case $e$ determines an embedding of $D W(9, q)$ into a subspace $\mathrm{PG}(131, q)$ of PG $(251, q)$. Similarly as in Lemma 4.6, we can show the following: 
Lemma 4.8. The subspace $\mathrm{PG}(131, q)$ of $\mathrm{PG}(251, q)$ is described by the following 120 equations:

$$
(-1)^{\sigma\left(K \backslash H_{1}\right)} X_{\left(K \backslash H_{1}\right) \cup H_{2}^{\prime}}=(-1)^{\sigma\left(K \backslash H_{2}\right)} X_{\left(K \backslash H_{2}\right) \cup H_{1}^{\prime}}
$$

for all subsets $H_{1}$ and $H_{2}$ of $K$ satisfying $\left|H_{1}\right|=\left|H_{2}\right|$ and $H_{1} \neq H_{2}$, and

$$
\begin{array}{ll}
X_{3,4,5,8,9}+X_{2,3,5,7,8}+X_{2,4,5,7,9}=0, & X_{3,4,5,8,10}+X_{2,4,5,7,10}=X_{2,3,4,7,8}, \\
X_{2,3,5,7,10}+X_{2,3,4,7,9}=X_{3,4,5,9,10}, & X_{2,4,5,9,10}+X_{2,3,4,8,9}+X_{2,3,5,8,10}=0, \\
X_{1,4,5,9,10}+X_{1,3,4,8,9}+X_{1,3,5,8,10}=0, & X_{4,5,6,9,10}+X_{3,4,6,8,9}+X_{3,5,6,8,10}=0, \\
X_{4,5,7,9,10}+X_{3,4,7,8,9}+X_{3,5,8,7,10}=0, & X_{2,4,7,8,9}+X_{2,5,7,8,10}=X_{4,5,8,9,10}, \\
X_{3,5,8,9,10}+X_{2,5,7,9,10}=X_{2,3,7,8,9}, & X_{3,4,8,9,10}+X_{2,3,7,8,10}+X_{2,4,7,9,10}=0 .
\end{array}
$$

One can now easily find ordered bases of $V(132, q)$ consisting of 32 vectors of weight 1,80 vectors of weight 2 and 20 vectors of weight 4 . One can take such an ordered basis, such that the bilinear form is represented by the matrix

$$
\operatorname{diag}(\underbrace{M_{1}, \ldots, M_{1}}_{16 \text { times }}, \underbrace{M_{2}, \ldots, M_{2}}_{40 \text { times }}, \underbrace{M_{6}, \ldots, M_{6}}_{5 \text { times }}),
$$

with $M_{1}$ and $M_{2}$ as before and

$$
M_{6}=\left[\begin{array}{rrrr}
0 & 0 & 4 & 2 \\
0 & 0 & 2 & 4 \\
-4 & -2 & 0 & 0 \\
-2 & -4 & 0 & 0
\end{array}\right]
$$

E.g., the following four linearly independent vectors of $V(132, q)$ give rise to the matrix $M_{6}$ :

$$
\begin{aligned}
& e_{3,4,5,8,9}+e_{1,2,5,6,7}-e_{2,4,5,7,9}-e_{1,3,5,6,8}, \\
& e_{2,3,5,7,8}+e_{1,4,5,6,9}-e_{2,4,5,7,9}-e_{1,3,5,6,8}, \\
& e_{3,4,8,9,10}+e_{1,2,6,7,10}-e_{2,4,7,9,10}-e_{1,3,6,8,10}, \\
& e_{2,3,7,8,10}+e_{1,4,6,9,10}-e_{2,4,7,9,10}-e_{1,3,6,8,10} .
\end{aligned}
$$

From the previous discussion, the following result readily follows.

Theorem 4.9. - If $q=2^{r}$, then $D W(9, q) \cong D Q(10, q)$ and the minimal polarized full embedding of $D W(9, q)$ is the spin embedding (vector dimension 32);

- If $q=3^{r}$, then the minimal polarized full embedding of $D W(9, q)$ has vector dimension 122;

- If $2^{r} \neq q \neq 3^{r}$, then the Grassmann embedding of $D W(9, q)$ (of vector dimension 132) is the unique polarized full embedding of $D W(9, q)$. 
The case $q=3^{r}$

We have shown in Theorems 4.7 and 4.9 that the Grassmann embedding of $D W(2 n-$ $\left.1,3^{r}\right), n \in\{4,5\}$, is not isomorphic to the minimal embedding of $D W\left(2 n-1,3^{r}\right)$. We will now show that this property holds for any $n \geq 4$. We start with the following property of Grassmann embeddings which holds for any prime power $q$.

Proposition 4.10. Let e denote the Grassmann embedding of $\Delta=D W(2 n-1, q)$. Then for every convex subspace $F$ of $\Delta$ of diameter at least $2, e_{F}$ is isomorphic to the Grassmann embedding of $F$.

\section{Proof:}

(1) Suppose first that $F$ is a max of $\Delta$ determined by the point $x_{F}$ of $W(2 n-1, q)$. We will use the same notation as in the beginning of this section. Without loss of generality, we may suppose that $x_{F}=(1,0, \ldots, 0)$. A point $x$ of $F$ is generated by the rows of a matrix of the following form:

$$
\left[\begin{array}{cccccccc}
1 & 0 & \cdots & 0 & 0 & 0 & \cdots & 0 \\
0 & x_{1,1} & \cdots & x_{1, n-1} & 0 & x_{1, n} & \cdots & x_{1,2 n-2} \\
0 & x_{2,1} & \cdots & x_{2, n-1} & 0 & x_{2, n} & \cdots & x_{2,2 n-2} \\
\vdots & \vdots & \ddots & \vdots & \vdots & \vdots & \ddots & \vdots \\
0 & x_{n-1,1} & \cdots & x_{n-1, n-1} & 0 & x_{n-1, n} & \cdots & x_{n-1,2 n-2}
\end{array}\right]
$$

where the $(n-1) \times(2 n-2)$-matrix $A$ with entries $x_{i, j}$ satisfies

$$
A \cdot\left[\begin{array}{cc}
0_{n-1} & I_{n-1} \\
-I_{n-1} & 0_{n-1}
\end{array}\right] \cdot A^{T}=0 .
$$

Writing down the Grassmann coordinates for the point $x$, we easily see that the restriction of $f$ to $F$ defines an embedding of $F$ which is isomorphic to the Grassmann embedding of $F$.

(2) Suppose now that $F$ has diameter less than $n-1$. Then consider a chain $F=F_{0} \subseteq$ $F_{1} \subseteq \cdots \subseteq F_{k}=\Delta$ of convex subspaces of $\Delta$ such that $F_{i}, i \in\{0, \ldots, k-1\}$, is a max of $F_{i+1}$. By downwards induction on $i \in\{0,1, \ldots, k\}$, we easily see that every embedding $e_{F_{i}}, i \in\{0, \ldots, k\}$, is isomorphic to the Grassmann embedding of $F_{i}$.

Corollary 4.11. Let $\Delta$ be the dual polar space $D W(2 n-1, q)$ with $n \geq 4$ and $q=$ $3^{r}$. Then the minimal full polarized embedding $e$ of $\Delta$ is a proper projection of the Grassmann embedding.

Proof: Let $F$ denote a convex subspace of diameter 4 . Then by Theorem 1.6, $e_{F}$ is isomorphic to the minimal full polarized embedding of $F$. By Theorem 4.7, $e_{F}$ is not Springer 
isomorphic to the Grassmann embedding of $F$. By Proposition 4.10, it now follows that $e$ is not isomorphic to the Grassmann embedding of $\Delta$.

Acknowledgements The authors wish to thank the referees for their valuable suggestions.

\section{References}

1. R.J. Blok and A.E. Brouwer, "The geometry far from a residue." Groups and Geometries (Siena, 1996), 29-38, Trends Math., Birkhäuser, Basel, 1998.

2. A.E. Brouwer and H.A. Wilbrink, "The structure of near polygons with quads," Geom. Dedicata 14 (1983), 145-176.

3. F. Buekenhout and P.J. Cameron, "Projective and affine geometry over division rings," Chapter 2 of the Handbook of Incidence Geometry F. Buekenhout (Ed.), Elsevier, Amsterdam, 1995.

4. F. Buekenhout and C. Lefèvre-Percsy, "Generalized quadrangles in projective spaces," Arch. Math. (Basel) 25 (1974), 540-552.

5. B.N. Cooperstein, "On the generation of dual polar spaces of unitary type over finite fields," European J. Combin. 18 (1997), 849-856.

6. B.N. Cooperstein, "On the generation of dual polar spaces of symplectic type over finite fields," $J$. Combin. Theory Ser A 83 (1998), 221-232.

7. B.N. Cooperstein and E.E. Shult, "A note on embedding and generating dual polar spaces," Adv. Geom. 1 (2001), 37-48.

8. B. De Bruyn and A. Pasini, "Minimal Scattered Sets and Polarized Embeddings of Dual Polar Spaces," preprint.

9. K.J. Dienst, "Verallgemeinerte vierecke in projektiven Räumen.” Arch. Math. (Basel) 35 (1980), 177186.

10. J.W.P. Hirschfeld and J.A. Thas, "General Galois Geometries.” Oxford Mathematical Monographs. The Clarendon Press, New York, 1991.

11. A. Kasikova and E.E. Shult, “Absolute embeddings of point-line geometries," J. Algebra 238 (2001), 265-291.

12. A. Pasini, "Embeddings and expansions," Bull. Belg. Math. Soc. Simon Stevin 10 (2003), 585-626.

13. M.A. Ronan, "Embeddings and hyperplanes of discrete geometries," European J. Combin. 8 (1987), 179-185.

14. E.E. Shult, “On Veldkamp lines,” Bull. Belg. Math. Soc. Simon Stevin 4 (1997), 299-316.

15. E.E. Shult and A. Yanushka, "Near n-gons and line systems," Geom. Dedicata 9 (1980), 1-72.

16. J.A. Thas and H. Van Maldeghem, "Embeddings of small generalized polygons," to appear.

17. J. Tits, Buildings of Spherical Type and Finite BN-pairs, Springer L. N. in Math. 386, Berlin, 1974.

18. A. Wells, "Universal projective embeddings of the grassmannian, half spinor and dual orthogonal geometries," Quart. J. Math. Oxford 34 (1983), 375-386. 